\title{
CoLinK: Cooperative Knowledge Management for Engineering Teams
}

\author{
Michael Klingemann ${ }^{1}$ and Jürgen Friedrich ${ }^{2}$ \\ ${ }^{1}$ University of Bremen, Center for Computing Technologies, Germany, klingemann@tzi.de \\ ${ }^{2}$ University of Bremen, Center for Computing Technologies, Germany, friedrich@tzi.de
}

\begin{abstract}
Due to today's increasingly complex processes in planning and design, knowledge management (KM) is becoming a crucial factor of success in the engineering sector. In a participatory design project we created a prototype for a process oriented KM system (CoLinK), which allows engineers to jointly model projects with generic process descriptions, creating a virtual engineering community within the enterprise and beyond. During every project these process descriptions will be augmented with knowledge annotations. The CoLinK system includes a document as well as contact management module and lets users semantically link the corresponding information in the process context. With any finished project engineers can utilize the acquired knowledge to cooperatively improve the quality of the generic process descriptions.
\end{abstract}

Keywords: Knowledge management, Engineering, Cooperative work, CoLinK

\section{Introduction}

Knowledge Management (KM) in the engineering sector is becoming increasingly important and challenging. Due to global markets, companies have to face international competition and can, therefore, no longer focus on just national business. Dealing with international clients increases the required amount of knowledge (e. g. country specific requirements and regulations) and at the same time decreases the local availability of key players due to business travelling, which demands for a system that is accessible from anywhere via Internet connection. On the other hand, sharing knowledge not only improves the quality of engineering products but also enhances the quality of work of the engineers.

Our system analysis, which has been conducted in several iterations within four engineering companies, has shown that intensive knowledge exchange takes place 
within the cooperative solving of complex processes (e. g. discussing required changes to drawings). The key for successful knowledge management is seen by the participating companies in the ability to reuse existing project schemes for future engineering projects.

Based on the findings in our analysis we propose that knowledge acquisition in the engineering sector has to take place during the process execution. Knowledge exchange has to be facilitated by encouraging discussion about encountered obstructions and possible solutions. Storing this information in form of annotations in combination with links to related documents and contacts constitutes valuable process knowledge that can aid future projects. According to these assumptions we have created a system named CoLinK (Cooperative Linking of Knowledge), a knowledge management system for cooperative engineering teams.

\section{Knowledge management in engineering - a deficit analysis}

\subsection{Methods: Analysis and inclusion}

The subject of our analysis - knowledge transfer in engineering teams - requires a thoroughly chosen set of research methods: Engineering teams are complex social organizations. They have to cooperate in differentiated, dynamic, and vaguely determined work environments. The need for cooperation is twofold: First of all, the engineering projects are subdivided into processes which often are being performed by different engineers and which, therefore, need a high degree of horizontal cooperation. Secondly, they have to cooperate to organize the knowledge exchange between experienced and less experienced (younger) engineers. Each engineering project is different from the other. This is especially true in international engineering projects where legal frameworks, physical conditions, working attitudes etc. vary in a broad range. Also the team members themselves show a substantial degree of variation with respect to their level of qualification, professional specialization, work experience etc. Analysis and design of work organization and IT infrastructures in such an engineering environment require a set of concerted methods beyond traditional standardized tools like multiple-choice questionnaires.

The overall approach we followed was taken from the Participatory Design paradigm which is quite popular in computer science $[1,2,3]$ : The IT development process, from analysis and design to implementation and testing, is no longer understood as a single sequence of steps following each other ("waterfall model") but as a repeatedly traversed cycle of development steps whereat each cycle results in a more elaborated prototype of the system ("evolutionary model"). Participatory design is especially useful in an area where engineering, planning or consulting tasks have to be performed [4]. 
To comply with the complex requirements of analyzing engineering work we have to deal with the problem "that users often are not sufficiently able to reflect their work situation and to express it appropriately in interviews. Users often do not have an adequate conceptual understanding of their role and behaviour in a work flow; many of their working methods are internalised and cognitively automated. Explaining those methods is difficult or even impossible for them." [2] This is what Polanyi described by the term 'tacit knowledge' [5] and what others call "tacit knowing" to indicate genuine dependency of this kind of knowledge on the human being. We tried to overcome this lack of explicitness by performing intensive in-depth talks with the engineering personnel - using a semi-structured guideline - as well as observing their task performance and communication behaviour at the workplace. Talks were performed with engineers, project managers, market researchers, external users, and the management. Conversations took up to four hours depending on the subject discussed. Topics of interest were:

- the organizational structure of the enterprise,

- the communication structure

- basic operational knowledge entities: projects, processes, tasks, documents (contracts, drawings), annotations, contacts (employees, supplier, experts) etc.

- kinds of knowledge, processes with special knowledge needs

- the formal and informal information flow

- knowledge exchange between local team members as well as with remotely operating members

- weaknesses in knowledge generation and distribution, and

- the IT infrastructure in use.

The analysis has been done in four different engineering companies, all of them SME with about 10 to 50 employees. The companies' profiles are as follows:

- Company A is planning huge chemical and pharmaceutical facilities as well as steelworks in several countries worldwide. The company runs branches in industrialized as well as developing countries and has to manage knowledge flows between engineers of hundreds of construction firms and suppliers.

- Company B is not only planning complex electrical and electronic installations and process controls for food industry, environmental technology and materials handling, but is also active in producing, assembling and maintaining control facilities for these businesses.

- Company $\mathbf{C}$ is a construction firm which mainly focuses on mechanical engineering as well as ship building industries (from passenger liners and cargo ships to container and navy vessels). Engineers in the large CAD departments provide the drawings which create the knowledge links to their customers.

- Company D is an engineering company specialized in the field of steel construction for electro-hydraulic engineering. Roll-on-roll-off ramps, quay facilities, bridge and sluice hydraulics as well as associated services characterize this company. 
The companies have been chosen to cover a broad range of engineering tasks which enables us to compare knowledge demands in different engineering areas and maybe get some general findings which can be used for tailoring the organizational and technical support structure for engineering knowledge work.

Participatory design in the development phase meant that we discussed each prototype with the engineering staff and implemented their proposals in a feedback loop. To facilitate prototyping we used rapid development tools allowing us to produce "throw-away prototypes" which could be used by the engineers handson without complete functionality. Meanwhile the system is transferred to an evolutionary prototype which is already in use in the participating companies. Next step will be to evaluate the CoLinK prototype in real project environments of the partner companies. We are just starting to develop appropriate evaluation methods $[6,7]$.

\subsection{System analysis: Empirical findings}

The following paragraphs describe the aspects discovered during the system analysis that had major influence on the design of the CoLinK system.

\section{Methodological competence of engineers}

In the engineering sector the execution of processes requires methodological competence which is important for quality assurance as even small mistakes during the process can be critical. This makes it particularly hard for young engineers who are not used to the details of the company's methods.

To target this problem one of our partner companies attempted to build up a "knowledge library" on the central file server where employees could store process relevant documents, e. g. process descriptions, guidelines, etc. Since the complex processes require mainly implicit knowledge, they did not succeed in exploring and storing that knowledge.

While these problems can be generally approached with document management software, we discovered further aspects that require an approach that goes beyond document management. Within the interviews (with the management) it showed that while the general processes itself are similar in many projects, they have their particularities depending e. g. on the type of facility or the country where it is constructed. Accordingly, to make use of any stored information not only a project reference is required (e. g. meta-data) but also information about the project itself to identify similar projects and make use of information that is connected to it.

Further more, the need to link contact information to processes, e. g. to identify experts, was discovered, which was so far done in a separate database with only rudimentary functionality and no link to either projects or processes. 


\section{Project traceability}

The traceability of projects is seen as an important factor by the participating companies and employees. Next to project oriented storage of emails on an exchange server, they attempted to log file transfers between different parties by putting copies of up/downloaded files into corresponding inbound/outbound folders on their file server. Having traceability for data exchange and correspondence partly in place, our partners lack support for tracing especially knowledge relevant project information. Even though they realized the importance of preserving knowledge creation and exchange within the processes they could not find a way to successfully implement it.

\section{Distributed knowledge management}

Due to business engagement in emerging markets e. g. in Asia and Eastern Europe staff members need to travel and are not always locally available. However, local presence is currently a key element for successfully conducting the engineering projects. To handle distribution, so far only VPN connection for remote file access and VoIP software is being used, however, this enables distributed knowledge exchange only to a certain extent. During the system analysis the high demand for distributed knowledge management became obvious. Since our partners are planning to extend the distributed work by installing subsidiaries in other countries to have a closer contact to their customers, support for distributed knowledge management becomes a central aspect for the CoLinK system.

\section{Information technology infrastructure}

The system analysis revealed a general lack of IT infrastructure in the participating medium sized companies, e. g. a document management system as a foundation for knowledge management was not used by any of our partners. Also existing standard components for shared email and calendar functionality and a central file server were not used to their full potential. While this situation caused the engineers to report many "standard problems" during our interviews, which complicated a clear focus on knowledge management issues in the beginning, it also enabled certain beneficial aspects:

- The importance of including information management techniques as a foundation for successful knowledge management was pointed out.

- Missing use of document management systems gave us clear evidence that including a DMS into the CoLinK system was a must.

The question arises how to bridge the gap between traditional information processing and future knowledge management by the use of adaptive ICT. 


\section{State of the art}

\subsection{Knowledge management and cooperation}

In the context of CoLinK we have to consider two dimensions of knowledge and knowledge management: a) Knowledge as an individual property of humans vs. a collective resource of organisations. b) Knowledge management as a technology to extract knowledge from humans vs. a cooperative approach to organise knowledge within institutions.

Knowledge as an individual property - a research subject of cognitive psychology - deals with topics like the organisation of the brain, different kinds of knowledge representations, properties of short and long term memory, problem solving etc. The second interpretation of knowledge extends the view from the individual to the organisation: How is knowledge created, stored and exchanged in organisations? The concepts of "learning organisations" and "organisational memory" are widely accepted as models for explaining creation, acquisition, integration, distribution and use of knowledge in organisational contexts [8, 9, 10]. Knowledge as a social category describes the background of the CoLinK cooperation model.

Reviewing knowledge management literature, on the one hand KM is seen as a technology to extract, organize and save human knowledge - mainly by means of artificial intelligence - in computer systems (data mining). The goal is to systematically retrieve this knowledge by standardized methods from the centralized knowledge repository and to apply it to a given problem. On the other hand KM is described as a socio-technical system which comprises the knowledge worker, the working environment, the social activity of knowledge exchange, the individual or collaborative problem solving process as well as the information technology to support these processes. In this understanding KM is not a technology to extract knowledge from humans but to multiply knowledge by sharing it between humans (see also [11]). This second approach which provides the basis of our project strongly relies on a number of assumptions which have to be considered when implementing the CoLinK system. To mention only some:

- Knowledge always depends on the organisational context of its generation and use.

- Knowledge is subjective in a way that it depends on its creator or bearer. Dreyfus and Dreyfus [12] defined five stages of expertise from beginner to expert each of which can be described by special characteristics of the respective level of knowledge.

- Knowledge often has a tacit dimension (see above). Knowledge workers have difficulties in explicitly communicating this kind of knowledge to co-workers.

- Knowledge is distributed among team members. This raises the question of the relation between centralized and decentralized knowledge.

Providing support for coping with these problems is the challenge the CoLinK system has to face. 


\subsection{Knowledge management in engineering}

Engineering constitutes the early stage of manufacturing, i. e. planning, conceptioning, requirements specification, construction, detailing of plans etc. It is a service provided as a crucial precondition to enable physical production. Therefore, knowledge management in engineering may be the key to a successful production management [13].

The reasons for developing KM strategies in industry and especially in engineering are: a) to preserve given and generate new knowledge within the company and b) to capture knowledge from outside the company and thereby strengthen the innovative power of the enterprise. Several instruments can be used to reach this goal: Exploring innovative knowledge sources, e. g. by cooperating with science and technology institutes. Not less important: using everyday liaisons with external partners like customers, subcontractors or consulting experts. The necessity of using these external knowledge sources often is underestimated especially by small and medium enterprises. Edler [14] proved this assumption in his case study about knowledge management strategies in German industry. The important role of knowledge intensive business services for the promotion of city regions as analyzed by Simmie and Strambach [15] underline these findings as well. The question is how to support this crucial success factor by an appropriate contact management component in the CoLinK system as well as a method to deal with distributed knowledge in internal and in externally related "communities of practice" [16].

\subsection{ICT support for knowledge management in engineering}

Considering the broad functionality of KM systems there are no common overall software systems targeting existing heterogeneous approaches. Because knowledge gathering, consolidating and exchange build up the centre of knowledge management, $C S C W$ and groupware systems are most often mentioned with first priority. More than 20 years of research in this field $[17,18]$ shows a great potential for applying theories and practices of CSCW in the fields of engineering and design. There are several interesting approaches for applying groupware to engineering tasks, showing advantages and problems. Pipek and Wulf [19] tried to install the Answer Garden approach - originally designed by Mark Ackerman and Thomas Malone [20] - in the environment of maintenance engineering in a steel mill. Results showed that division of labour, competition between engineers, and rivalry between organisational units often obstruct effective cooperation within the Answer Garden framework which hardly can be addressed by groupware technology. Perry [21] applies CSCW and groupware to design activities in construction engineering. As a result of two case studies (design work in civil engineering, con- 
sulting engineers in building design) he stresses the fact that CSCW systems in engineering often consider organisations as stable units neglecting social dynamics and organisational change. In our approach we try to cope with this problem by providing a high degree of flexibility and perspectivity as properties of CoLinK.

Secondly, document management systems are a strong basis to build upon: Documents in most cases can be seen as "information", e. g. a drawing of a pump in mechanical engineering normally does not contain knowledge about the context of use (e. g. climate) or about dismantling for repair. Therefore, the semantics of document management systems has to be enriched by the pragmatics of use. One possibility to reach this higher level of semiotics is to add meta-data to the basic information.

Thirdly, in contrast to information, knowledge in most cases is connected to processes. Therefore, in enterprise environments project management systems serve the dynamic side of knowledge management.

The number of software houses providing components for knowledge management is huge. They often present an impressive range of features from a content library, a workbench, different discussion boards up to a task manager which allows project managers to assign tasks to team members and to keep a log of current status of each task. Most of the systems aim at big companies; the requirements of small and medium enterprises are often not in the focus of these systems: not appropriate, too big, too difficult to use, too expensive. The CoLinK system tries to meet the requirements of these smaller firms and to integrate necessary functions to an easy to use configurable, scalable and affordable KM system.

\section{CoLinK system experience}

\subsection{General approach}

The conducted interviews and observations revealed that it would not be helpful to concentrate on single aspects like document, project or contact management. Certainly, within each segment an improvement could be reached by extending the existing solutions, however, the highest potential for improved information and knowledge exchange was seen in the linking of these aspects within the project context.

Thereby, it was understood as a critical success factor that the system does not aim at replacing existing technologies but to seamlessly integrate into the companies' infrastructure. For the contact management a bidirectional synchronization tool has been developed that let the partner companies continue to use their existing tools for contact management (e. g. Microsoft Outlook and proprietary databases). Since all partners are running common file servers in their networks with a consistent folder structure for their projects a document management system (Alfresco) was chosen that provides a standard network share to access the repository. 
Therefore, no extra client software has to be installed and the folder structure can be maintained while all benefits of the document management system are available to the CoLinK system.

To ensure intensive use of the system a major part of the knowledge collection takes place within the team members' task execution. The system, therefore, provides a "tasks" view where team members can not only see their assigned tasks but are also able to attach additional information like documents, contacts and annotations to it. The latter is intensively used for information/knowledge exchange between team members and can be seen as an integrated forum for each task allowing them for example to report problems and jointly find solutions for them. Next to annotating the task itself, a created link can be annotated as well, e. g. to explain the role of a company/person within a task.

Similarly to the "tasks" view, the "contacts" and "documents" view allows users to attach any of the earlier mentioned types of information to contacts/documents; however this paper focuses mainly on the functionality within the project context.

Information about projects as well as general project functionality like creating and editing projects is provided in the "projects" view, which lets users add project-members to the project, check project documents and model projects by creating and adding processes to the project.

Having this functionality in place, any information that was acquired during a project can later be evaluated and used for future projects gaining efficiency as well as providing quality assurance.

\subsection{Interface design}

The CoLinK interface design consists of several views that offer support for the different actions. In each view the screen is separated into frames that hold the panels, which provide a distinct part of the view's functionality. For example the "tasks" view shown in figure 1 contains the "tasks" and the "info" panel. 


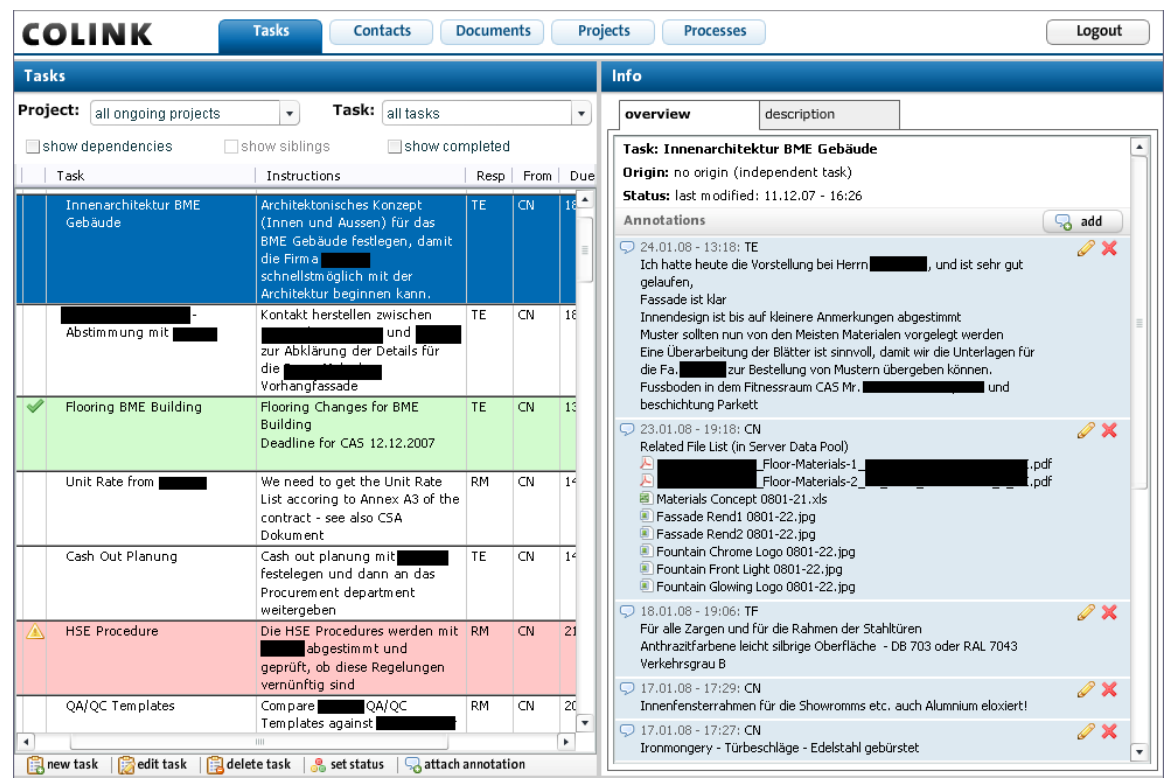

Figure 1. Tasks view with two panels

By clicking a task on the left side, detailed information is shown on the right side. This allows the user to browse through the tasks without losing the context. Similar to actual browser frames the frames in CoLinK can be resized, causing the embedded panels to adjust to the new size. This allows users with a higher resolution to benefit from the extra space. For many actions in CoLinK (e. g. "new annotation" or "create new project") popups are used to avoid switching to a new screen, which could distract users from their current "position" in the system.

\subsection{Processes vs. tasks}

A major requirement of our project partners was the possibility to create generic process descriptions for common actions within a project. These descriptions serve as guidelines and are especially useful for young engineers as they provide important knowledge about how the company works.

The description of the processes is comparable to Wiki articles; however, the CoLinK processes differ in a way that (just like explained earlier for the tasks) contacts, documents and annotations can be attached.

While these general processes can often be reused in several projects (see "modelling projects" in 4.4) the actual execution differs depending on the type of project. Therefore, the processes (i. e. the description and the additional information) have to be adapted to the individual project. To enable this, CoLinK imple- 
ments a versioning system that keeps track of the processes being used in the different projects.

To connect the processes to the task management it is possible to assign a process to a user, which will make the process appear in the corresponding task list.

Since a complex project cannot be described only by generic processes CoLinK offers different types of processes within a project:

- Generic processes are created in the "processes" view and stored in the process library. They are added to projects from the library and can then be modified within the project.

- Project specific processes can be created independently from the library within the project modeler. They are especially useful for processes that are expected to be only used in one project or processes that are used for the first time and cannot yet be generically described.

- Tasks can be created directly within the "tasks" view and are expected to be less complex and not easily reusable in future project. They are, therefore, only shown in the task list and not in the project modeler.

While the initial idea for the process handling was to create generic processes and then adapt them to the project needs, results from the companies use of the system show that so far they almost only used project specific processes to model their projects. It is assumed that it is easier for them to first create a process for an actual project and derive generic processes afterwards.

\subsection{Modelling projects}

To exploit the similarity of projects and make use of information and knowledge that was created in former projects, it is necessary to set certain parameters for every new project. Since companies specify their projects differently, these parameters can be configured to fit the companies' needs (e. g. country, project type, facility type, etc.). In a next step processes are added to the new project frame, which can be either done from the process library or from existing projects. Due to the fact that processes in the engineering sector are usually complex it is possible to create sub processes which are displayed in a hierarchical list.

Since it is the aim of the CoLinK system to embed knowledge management in the everyday work as proposed by Hoffmann et al. [22] project managers can assign the project processes to users who can then add information to them during the actual tasks execution (see 4.5). The project management functionality is very limited (e. g. assigning due dates and setting task status) and is not supposed to replace existing project management systems. In further versions of CoLinK we may consider implementing an interface for integration of existing project management tools. 


\subsection{Contextual linking and annotation}

The acquisition of project specific knowledge is done within the execution of tasks to minimize the required effort. Since engineers regularly use the CoLinK system to view, select and set the status of their tasks, any information can easily be added within the process context. By selecting a task, related information is shown, which includes the process description, related documents, contacts and hyperlinks. By integrating a document as well as contact management system, users can easily link any of the mentioned artefacts to the task. Unlike the generic process descriptions, which require a collaborative approval process, changing the description within a project can be done directly by the person who is currently working on the task.

A central part of the knowledge acquisition is the use of categorized annotations, which are attached to the task. These categories allow for example to report obstructions that require the help of other engineers. To encourage the collaborative finding of solutions, any task that is marked with an obstruction is visible to all other engineers, enabling them to reply to the reported obstruction.

With contextual links and annotations being the major means of knowledge management in the CoLinK system, we realized that in fact a combination of both enables interesting possibilities to foster knowledge acquisition, therefore.

- any annotation can include links to other artefacts and

- any link can be augmented with annotations

The application of the above mentioned cases can be illustrated with the following examples:

1. An obstruction within a task is reported with an annotation that includes a link to the corresponding document (e. g. problem within a drawing).

2. A contact is linked to a task with an annotation that explains the connection between the contact and the task.

To give users the chance to attach information in multiple steps, created links and annotations can be modified and enhanced with further information at any time.

\section{Implementing the CoLinK system}

The CoLinK system is designed to link different types of information within a project/process context. To enable the integration into existing infrastructure it aims at using enterprise content management (ECM) components and connecting them to the CoLinK core system. So far this is implemented for the open source document management system Alfresco, which is integrated via web services; however, the use of other systems is generally possible. 
The CoLinK core system consists of a database driven process management that also takes care about the versioned linking of the various kinds of information. Processes can have an unlimited number of hierarchies and also store information about their origin. This enables the required traceability when processes are copied and modified within the same or between different projects. The database also holds tables for projects and the company specific configuration of project properties as well as tables for companies and persons with the corresponding relation. Furthermore tables for the versioned annotations exist. Linking is done in a very generic way, storing sourceType and sourceID as well as destinationType and destinationID, which allows any combination of linking between the different types of information. Similar to the processes, the origin is stored for the links as well to enable traceability for the linked information of project processes.

The CoLinK client is a web based system using Web 2.0 technology to provide a user experience that is otherwise only known from desktop applications. Currently the prototype is implemented in Adobe Flash, which allowed rapid prototyping due to many existing components. For client/server communication Flash Remoting is being used, which offers RPCs and great debugging functionality.

While the performance and general user experience with the Flash client has been received positively by our partners, several aspects let us consider changing to an AJAX implementation in the future. For example the following usability problems with the Flash client have been discovered: keyboard initiated copy and paste does not work properly with the Firefox Flash plug-in; browser search functionality does not work; browser plug-ins do not work (e. g. Skype plug-in for direct dialling from within the application); parallel use of Flash and HTML causes problems in some browsers. Especially the last point is an important factor as we plan to include existing HTML/JavaScript components (e. g. TinyMCE for editing process descriptions).

\section{Conclusion and perspectives}

Our approach of process oriented knowledge acquisition with contextual linking and annotation enables a simple and user friendly way of creating an organizational memory and provides engineers with the opportunity to benefit from the stored knowledge during their everyday tasks. The CoLinK system is being used successfully by our partner companies and continuous feedback is reported back to our research group.

The prototype already changed the organizational strategies in participating companies to an extent that distributed teams work together on tasks that used to be done only in a collocated manner. It has shown that connecting the system to the companies' existing infrastructure (e.g. contact management) was a critical success factor for introducing the system. 
While the user interface in general has been adopted well, further improvements are necessary, especially drag and drop functionality will be a major aspect.

Even though several types of additional information can already be linked, observation of the system usage has shown that including email attachments for the annotations would be a great feature.

By introducing the CoLinK system not just in one company we have the opportunity to compare the evaluation results from different viewpoints which will give further input for the development. It is expected that the results will bring up aspects of customization to adjust the system to the specific needs of the companies. The next step could then be a generalization of the approach to apply our concept for process oriented knowledge management also to companies outside the engineering sector.

\section{References}

1. Beyer, H., Holtzblatt, K.: Contextual design: defining customer-centered systems. Morgan Kaufmann Publishers, San Francisco (1997)

2. Friedrich, J.: Participatory prototyping. In: Rauner, F., Maclean, R. (Eds.), Handbook of Vocational Education Research. Springer Publishers, Dordrecht (2007)

3. Kensing, F., Blomberg, J.: Participatory design: issues and concerns. Computer Supported Cooperative Work, Vol. 7, pp. 167-185 (1998)

4. Gärtner, J.: Participatory design in consulting. Computer Supported Cooperative Work - A Journal of Collaborative Computing, Vol. 7, pp. 273-289 (1998)

5. Polany, M.: The tacit dimension. Routledge and Keel Paul, London (1966)

6 Neale, D.C., Carroll, J.M., Rosson, M.B.: Evaluating computer-supported cooperative work: models and frameworks. In: CSCW '04, Vol. 6, Issue 3, pp. 112-121, ACM, New York (2004)

7 Ramage, M.: The learning way: Evaluating co-operative systems. Ph.D. Thesis, Lancaster University, Lancaster (1999)

8 Argyris, C.: On organizational learning. Blackwell Publishing, Oxford/Malden (1999)

9 Schwartz, D.G., Divitini, M., Brasethvik, T. (Eds.): Internet-based organizational memory and knowledge management. Idea Group Publishing, Hershey (2000)

10 Ramage, M., Reiff, F.: Links between organisational memory and cooperative awareness. http://www.comp.lancs.ac.uk/computing/research/cseg/projects/evaluation/OM_CA.html. Accessed 1 Feb 2008.

11. Bonifacio, M., Bouquet, P., Traverso, P.: Enabling distributed knowledge management: managerial and technological implications. Informatik/Informatique, no. 1, pp. $23-29$ (2002)

12. Dreyfus, H. L., Dreyfus, S. E.: Mind over machine. Free Press, New York (1986)

13. Shankar, R., Singh, M. D., Gupta, A., Narain, R.: Strategic planning for knowledge management implementation in engineering firms. Work Study, Vol. 52, no. 4, pp. 190-200 (2003)

14. Edler, J.: Knowledge management in German industry. Fraunhofer Institute for Systems and Innovation Research (ISI), Karlsruhe (January 2003)

15. Simmie, J., Strambach, S.: The contribution of knowledge-intensive business services (KIBS) to innovation in cities: an evolutionary and institutional perspective. Journal of Knowledge Management, Vol. 10, no. 5, pp. 26-40 (2006)

16. Wenger, E.: Communities of practice. Learning, meaning and identity. Cambridge University Press, Cambridge (1998) 
17 Twenty years of $\mathrm{CSCW}$ - what have we learned? Plenary panel. CSCW '06, ACM, New York (2006)

18 ACM (Ed.): GROUP '07. Proceedings of the 2007 International ACM Conference on Supporting Group Work. ACM, New York (2007)

19. Pipek, V., Wulf, V.: Pruning the Answer Garden: Knowledge Sharing in Maintenance Engineering. In: European Conference on CSCW, Kluwer, Amsterdam, pp. 1-20 (2003)

20 Ackerman, M.S., Malone, T. W.: Answer Garden: A Tool for Growing Organzational Memory. In: Proceedings of the ACM Conference on Office Information Systems, pp. 31-39, (1990)

21 Perry, M.J.: Distributed cognition and computer supported collaborative design: The organisation of work in construction engineering. Ph.D. Thesis, Brunel University, Uxbridge (1997)

22. Hoffmann, M., Loser, K., Walter, T., Herrmann, T.: A design process for embedding knowledge management in everyday work. In: Proceedings of the Conference on Groupware, ACM, pp. 296-305 (1999) 